\title{
Mystik und gegenstandslose Malerei
}

\author{
Von SIXTEN RINGBOM
}

$\mathrm{Zu}$ den verschiedenen Aufgaben, denen sich das Donnersche Institut widmen will, gehört „,das Erforschen der Mystik und ihrer Manifestationen in der Kunst“. Mystik und Kunst umfassen ein sehr weites Gebiet; chronologisch und geographisch betrachtet umspannt es Phänomene von ost- und zentralasiatischen Kulturen über frühes Christentum und Mittelalter bis zur Emblematik der Renaissance und des Barocks und den visionären Phantasien der Romantik und des Symbolismus. Wir haben soeben einen Vortrag gehört über solche Züge bei William Blake.

Die Fragen, die ich in diesem Zusammenhang berühren möchte, hängen zusammen mit der Kunst der neuesten Zeit und zwar mit der abstrakten oder gegenstandslosen Malerei ${ }^{1}$. Im Gegensatz zu dem, was man gewöhnlich

\footnotetext{
${ }^{1}$ Die mystischen - oder genauer ausgedrückt - theosophischen Züge in der Theorie und Rechtfertigung der abstrakten Kunst sind von verschiedenen Verfassern verschieden aufgefasst worden. Im grossen und ganzen ist das Problem von der ästhetisierenden Schule ignoriert worden. Andere haben die Bedeutung solcher Einflüsse hervorgehoben, ohne auf Einzelheiten einzugehen, wie z. B.W. Grohmann (Wassily Kandinsky - Leben und Werk, 2. Aufl., Köln 1961, SS. 41, 54, 83 f., 87, ro8) und M. Seuphor (Piet Mondrian - Leben und Werk, Köln r 957, SS. 54, 56 ff.). Einflüsse der Theosophie werden ausdrücklich bestritten von K. C. E. Lindsay (An Examination of the Fundamental Theories of Wassily Kandinsky [ungedr. Diss.], Madison, Wis., 195 I, SS. 208-13) und P. Selz (,,The Aesthetic Theories of Wassily Kandinsky and their Relationship to the Origin of Non-Objective Painting ", Art Bulletin, 29, 1957, SS. 127-136). Weiter gibt es Verfasser, die die gnostischen Züge in der abstrakten Kunst nicht nur zugeben, sondern sie sogar anerkennen (z. B. C. Giedion-Welcker , Kandinskys Malerei als Ausdruck eines geistigen Universalismus", Das Werk, 37, r950, SS. II9-123), oder die in der Abstraktion das Heraufkommen einer neuen religiösen Bildform sehen und eine Wiedergeburt des ,,Meditationsbildes" (siehe H. Debrunner (hrsg.), Wir entdeckten Kandinsky, Zürich I947, SS. $x_{4}$ f., 27, 34, 53, 60 ff.; A. Rosenberg, Die christliche Bildmeditation, München 1955, S. 33; K. Ledergerber, Kunst und Religion in der Verwandlung, Köln 1961, SS. xo2 f.). Die okkulten Ahnen der modernen Kunst wurden von T. H. RobsjohnGibbings (Mona Lisa's Mustache, New York 1947) als eine Waffe gegen alle moderne Kunst benutzt. Robsjohn-Gibbings' skurriles Pamphlet enthält doch einige scharfe Beobachtungen. Ein mehr ausgewogener Kommentar zu diesem Problem findet sich bei Klaus Brisch, Wassily Kandinsky (1866-1944), Untersuchungen zur Entstehung
} 


\section{INVOLUTION \& EVOLUTION}

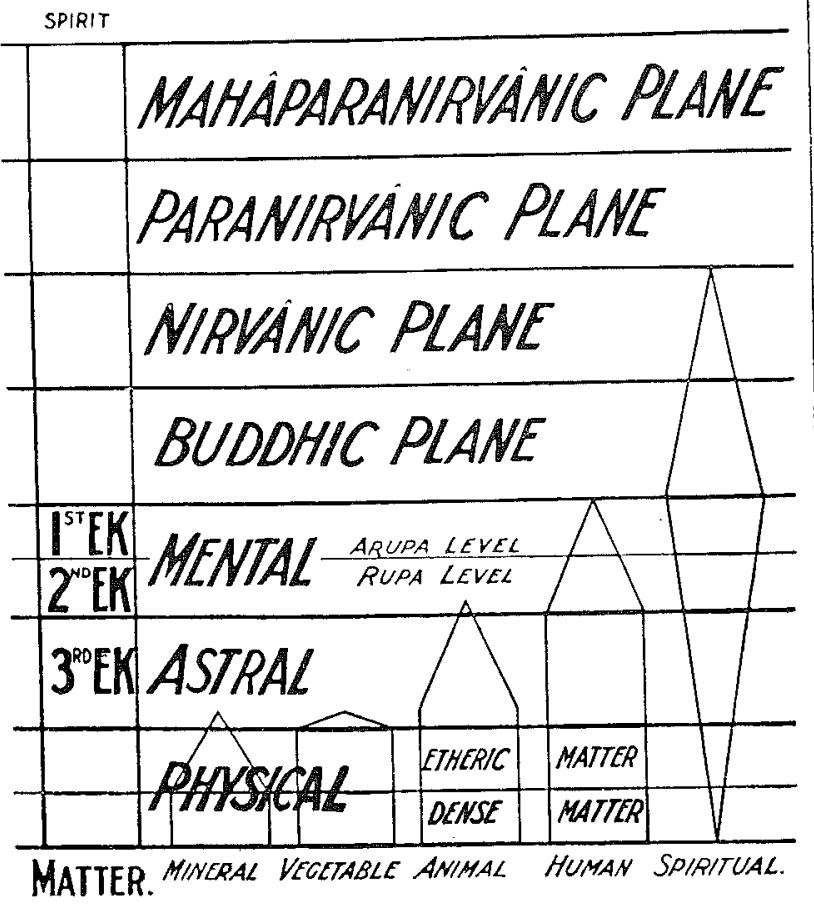

I. Aus Leadbeater, Man Visible and Invisible, Tafel IV.

der gegenstandslosen Malerei an seinem Werk von I900-I92I (ungedr. Diss.), Bonn 1955, SS. 329-331, und bei M. Seuphor, Knaurs Lexikon abstrakter Malerei, München \& Zürich 1957 , SS. 5o ff. Von neueren und mehr ausführlichen Behandlungen der Frage kann man die folgenden nennen: P. Fingesten, ,Spirituality, Mysticism and Non-Objective Art", The Art Fournal, 30: I, I96I, SS. 2-6; L. D. Ettlinger Kandinsky's , ,At Rest", London 1961, SS. 6, 8 f., 20.; H. L. Jaffé, De Stijl I9I7-3I, Der niederländische Beitrag zur modernen Kunst, Berlin x965, SS. 2 I f., 65-74, 122; und des Verfassers ,Art in the 'Epoch of the Great Spiritual': Occult Elements in the Early Theory of Abstract Painting", Fournal of the Warburg and Courtauld Institutes, 29, r966, SS. 386-418. Ich hatte keine Gelegenheit die ungedruckte Dissertation von Laxmi P. Sihare, Oriental Influences on Wassily Kandinsky and Piet Mondrian Igog-I9I7, New York University 1967 , zu benutzen, das zum Zeitpunkt der Niederschrift noch nicht für Mikroverfilmung freigegeben ist. Dr. Robert Welsh (University of Toronto) arbeitet, wie ich höre, an Mondrians Verhältnis zur Theosophie, und Miss Rose Carol Washton (The Guggenheim Museum, New York) arbeitet an einer Dissertation über Kandinsky die, wenn ich recht verstanden habe, diese Frage berühren wird. 


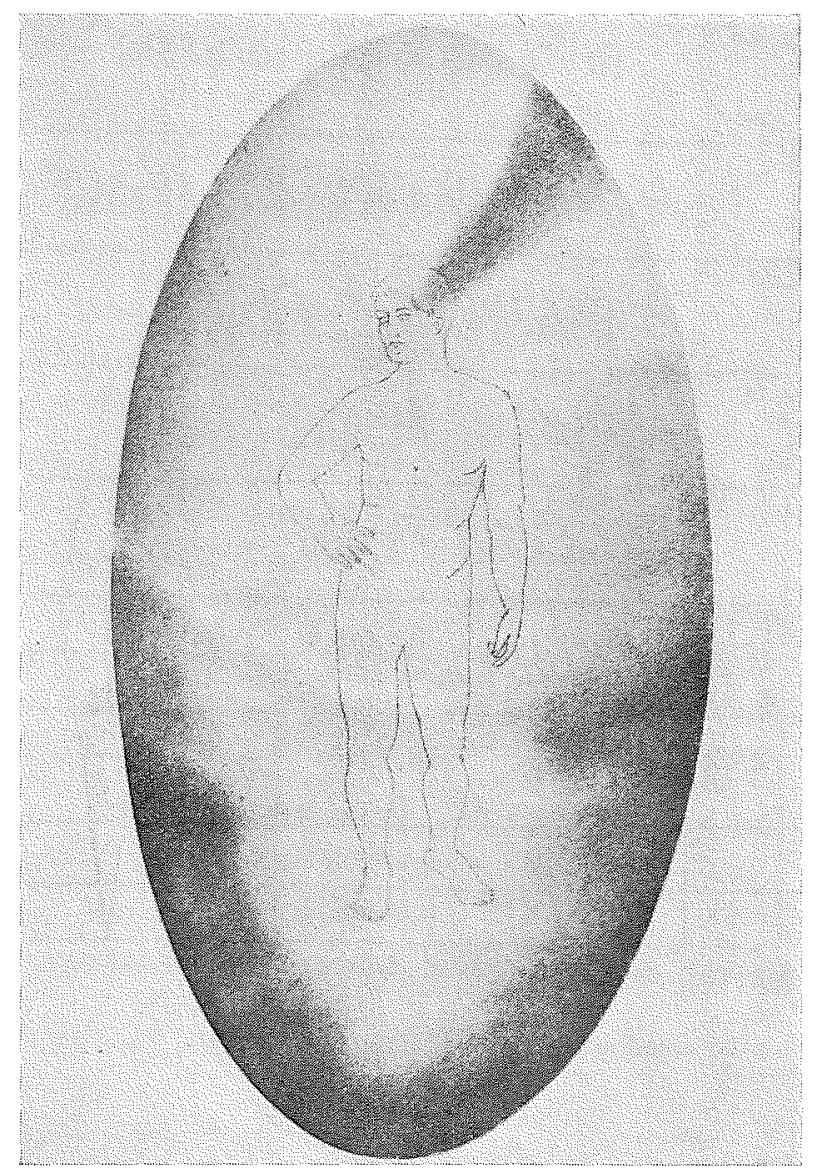

2. Mentalkörper. Aus Leadbeater, Tafel IX.

annimmt, zeigt es sich nämlich bei näherer Betrachtung, dass diese schon über ein halbes Jahrhundert alte Richtung ihre ursprünglichen Bedingungen nicht in einer rein ästhetisierenden Kunstbetrachtung oder einem auf die Spitze getriebenen Formalismus hat. Hinter dem Gedanken von dem nichts vorstellenden, gegenstandslosen Bild verbirgt sich vielmehr eine Theorie, die man sowohl ihrem Inhalt als ihrem Ursprung nach mit gewissen mystischen Anschauungen in Verbindung setzen kann. Um diese Behauptung zu begründen, werde ich meine Darlegungen nach drei Aspekten gliedern: (I) „Bildlosigkeit" oder „Formlosigkeit" in Mystik und Meditationspraxis; (2) "Gegenstandslosigkeit" in der abstrakten Kunst; (3) die Frage des tatsächlichen, historischen Zusammenhanges zwischen (I) und (2). 


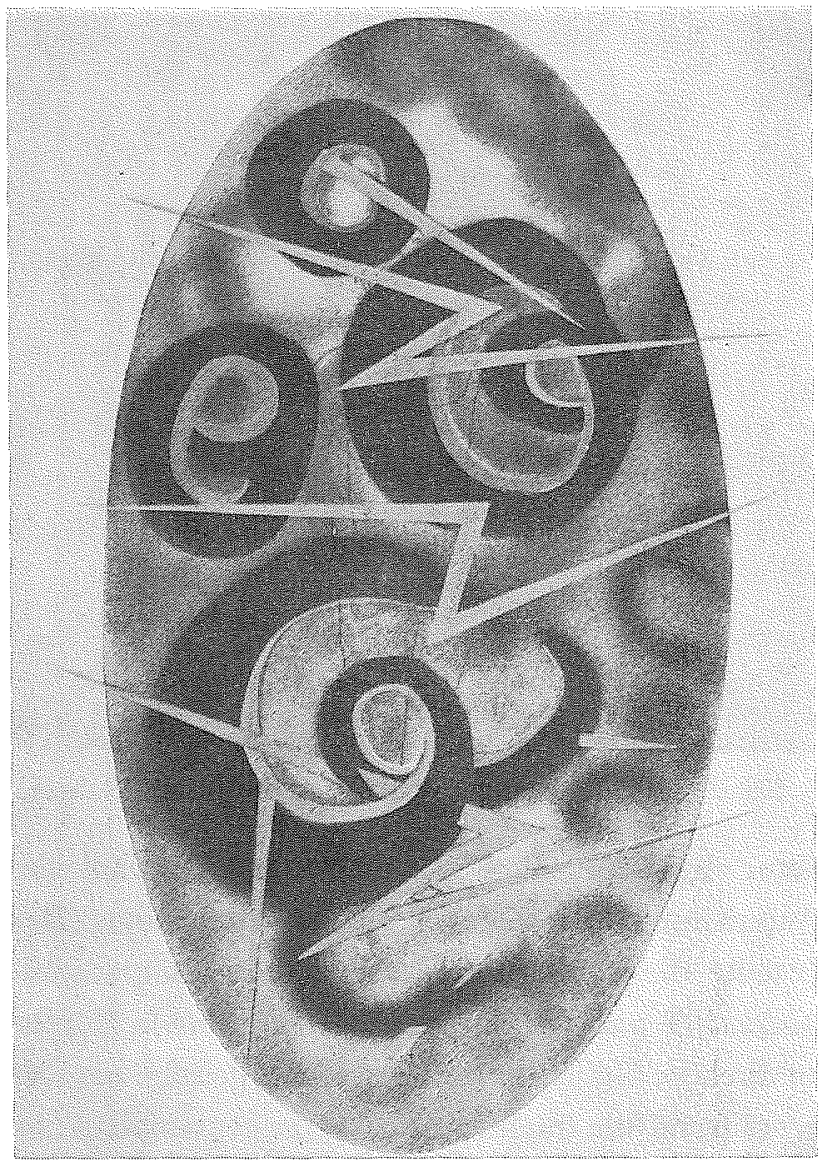

3. Astralkörper (Zorn). Aus Leadbeater, Tafel XIII.

\section{Bild- und Formlosigkeit in der Mystik}

Der Begriff „Bildlosigkeit“ oder „,Formlosigkeit“ bezeichnet in der Mystik einen Bewusstseinszustand, in welchem man sich von seinen Sinneseindrücken und Seelenbilder befreit hat. Dieser Anschauung liegt ein psychologisches Modell zugrunde, nach welchem die Seele als Empfänger wirkt für von aussen kommende Bilder der materiellen Dinge oder als ein Gefäss, in dem Seelenbilder spontan auftauchen. Verschiedene Formen dieser psychologischen Betrachtungsweise treten auf im Denken der Antike, z. B. bei Plato, Aristoteles und den Epikuräern; im Neuplatonismus macht sich eine Tendenz bemerkbar, den Seelenbildern sogar die Priorität vor der 


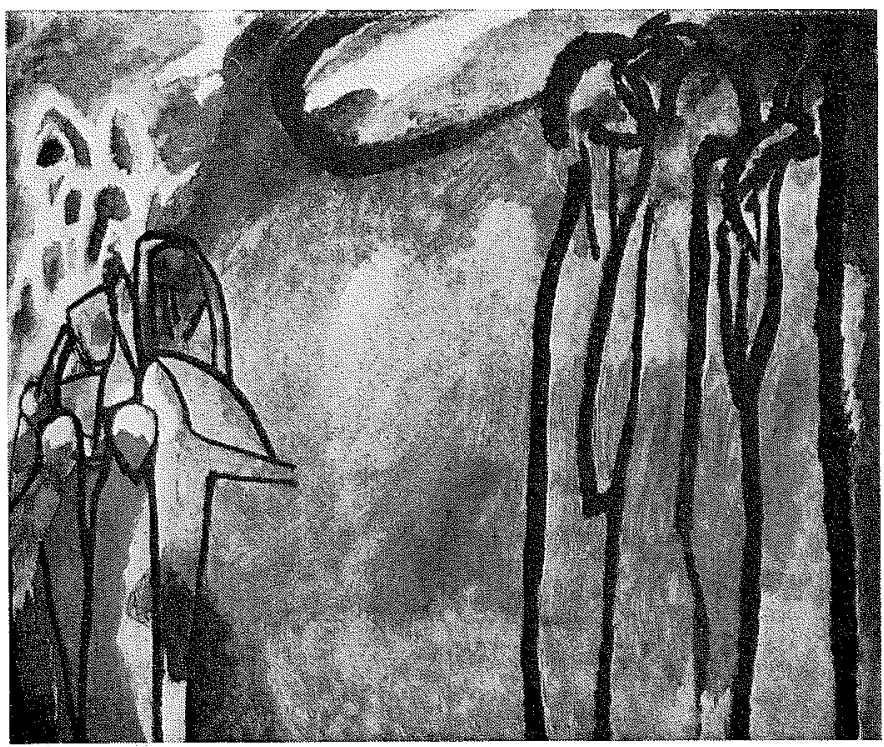

4. Wassily Kandinsky, Improvisation I9 (I9I r). Städtische Galerie, München.

äusseren Natur einzuräumen ${ }^{1}$. Für die christliche Psychologie war es eine Stelle bei Augustinus, die massgebend wurde. Augustinus (De Gen. ad Litt. XII, 4, II) teilte die Visionen ein in drei Arten: körperliche (corporates), geistige (spirituales) und intellektuelle (intellectuales). Körperliche Bilder entstehen an direkten Gesichtswahrnehmungen, geistige aus Erinnerung und Einbildung, während die intellektuelle Vision kontemplativ ist. Diese Einteilung findet sich auch im christlichen Neuplatonismus (Pseudo-Dionysios, De Eccl. Hier. I, 2) und bei Gregorius dem Grossen, welcher der Ansicht war, dass die Bildkunst ihre Aufgabe auf der untersten Stufe hätte: das körperliche Betrachten einer Malerei bedeutet, dass wir per visibitia invisibilia demonstramus (Ep. IX, 52).

Schon bei Plotinos taucht aber der Gedanke auf, dass sichtbare Formen ein Hindernis bilden für die Kontemplation, ein Hindernis, das man überwinden muss, wenn man bis zur Ekstase kommen will. Darum ist schon ein Seelenbild einem direkten Gesichtseindruck vorzuziehen, meint er in dem berühmten Beispiel mit dem Liebenden (Enn. VI, 7, 33). Aber das rein Form-

${ }^{1}$ A.-E. Chaignet, Histoire de la psychologie des Grecs, II, Paris I 889 (Nachdr. Brüssel I 966), SS. 329 ff.; über Neuplatonismus, siehe IV, Paris I892 (Nachdr. 1966), SS. I $69 \mathrm{ff}$. 


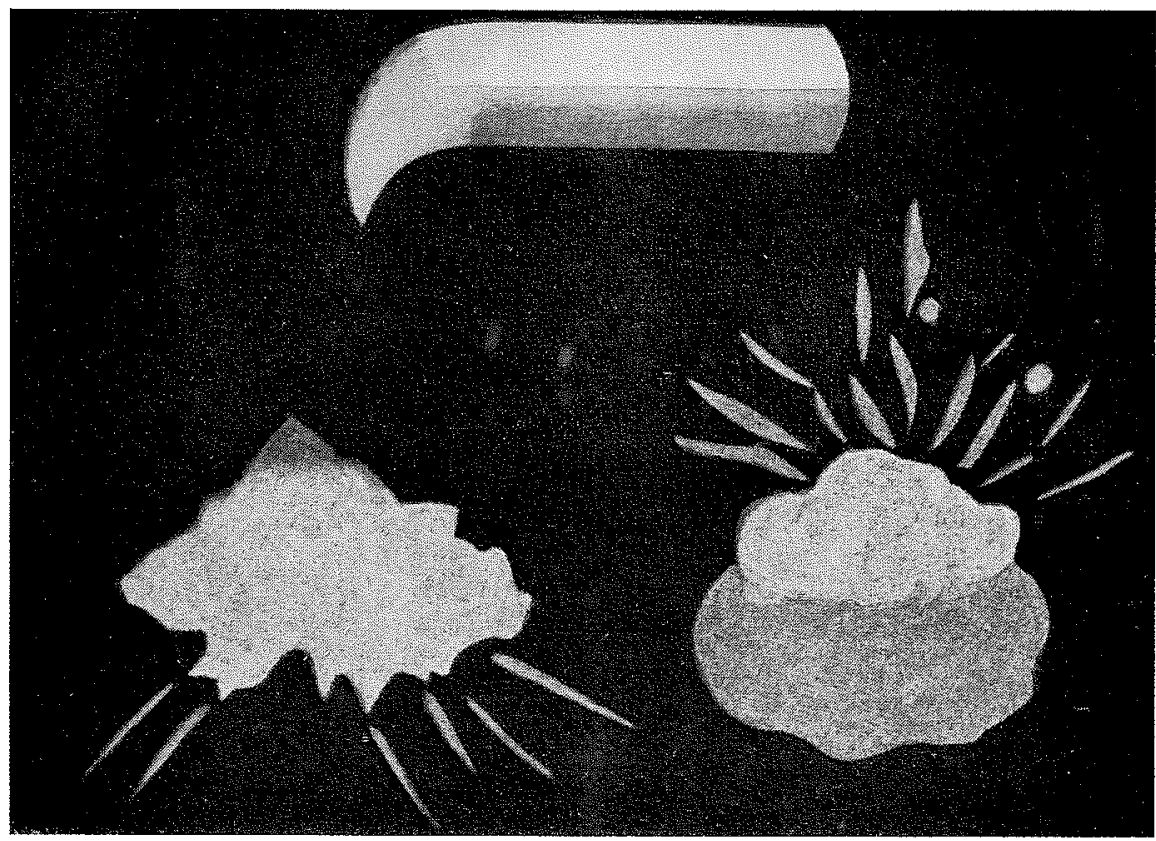

5. Bei einem Schiffbruch. Aus Besant und Leadbeater, Thought-Forms, Tafel 30.

lose, das sowohl von Sinneseindrücken wie Seelenbildern befreite Schauen bildet das höchste Stadium (Enn. VI, 7, 32f.). Das reine Schauen ist formlos - frei von sichtbaren Formen. Derjenige, der Gott schauen will, muss deshalb ,abstrahieren von allem“ (Enn. VI, 8, 2I).

In der mittelalterlichen Mystik wurde mit der Zeit gerade die Bild- oder Formlosigkeit als eine Bedingung für die Unio immer mehr betont. Der hl. Bernhard predigte z. B.: ,,.. du hast nichts erreicht, wenn du dich nicht durch die Reinheit des Sinnes befreit hast von den Wahnbildern der körperlichen Bilder, die von allen Seiten hereinströmen" (Cantica 52,5). Nach dem hl. Bernhard wurde diese Forderung immer wichtiger. Heinrich Suso vertrat die Ansicht, dass eine Vision umso edler ist ,,ie vernúnftiger und bildloser" ${ }^{\prime 1}$ sie ist, und er betrachtete diese ,bildlosekeit" ${ }^{\prime 2}$ als ein Ideal. Johannes Tauler rechnet in der augustinischen Tradition mit drei Arten von

${ }^{1}$ Heinrich Seuse, Deutsche Schriften, ed. K. Bihlmeyer, Stuttgart I907 (Nachdr. Frankfurt/M I961), S. I 83 .

2 Seuse, S. 97. 


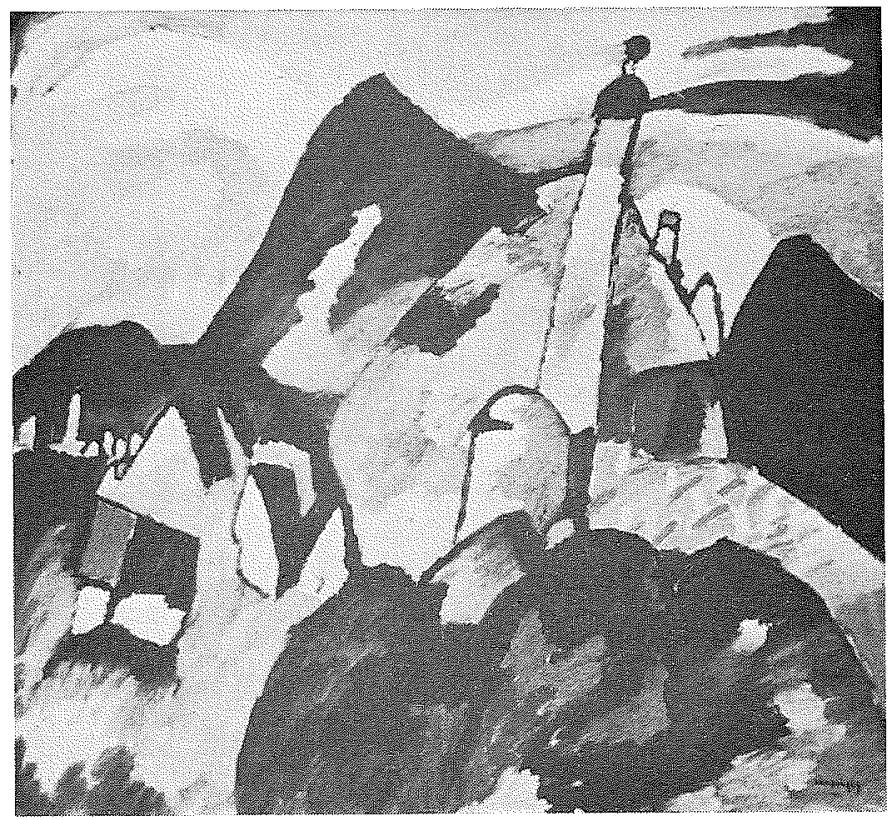

6. Kandinsky, Kirche in Murnau (г10), Stedelijk Van Abbe Museum, Eindhoven.

Schauungen; auf der dritten Stufe, ,vallent alle bilde und forme und glichnisse abe"

Die Beispiele könnten beliebig vermehrt werden, aber wir schliessen die Reihe mit einem aus dem späten Mittelalter. In der Nachfolge des hl. Bernhard erwähnt der Niederländer Hendrik Herp vier Hindernisse für die Kontemplation, wovon quartum impedimentum sunt phantasmata corporalium imaginum, quae se imprimunt cordi et difficillime possunt repelli. Durch das Ausschliessen der körperlichen Bilder kann doch ,,der Spiegel der Seele $^{\text {" zuletzt von Bildern gereinigt werden }}{ }^{2}$.

Diese Ansicht prägt auch die spätmittelalterliche, auf Gregorius fussende Bildtheologie. So nahm z. B. Jean Gerson eine tolerante Haltung gegen das Kunstwerk als Hilfsmittel bei der Meditation ein, hob aber gleichzeitig hervor „dass wir lernen müssen, uns mit unseren Sinnen über die sichtbaren

${ }^{1}$ Johannes Tauler, Die Predigten, hrsg. F. Vetter (Deutsche Texte des Mittelalters, I I), Berlin I9ro, S. 2 I.

${ }^{2}$ Henticus Harph, Theologia mystica, Köln I 538 (Nachdr. Farnborough 1966), fol. $\mathrm{cli}^{\mathrm{v}} \mathrm{ff}$. 


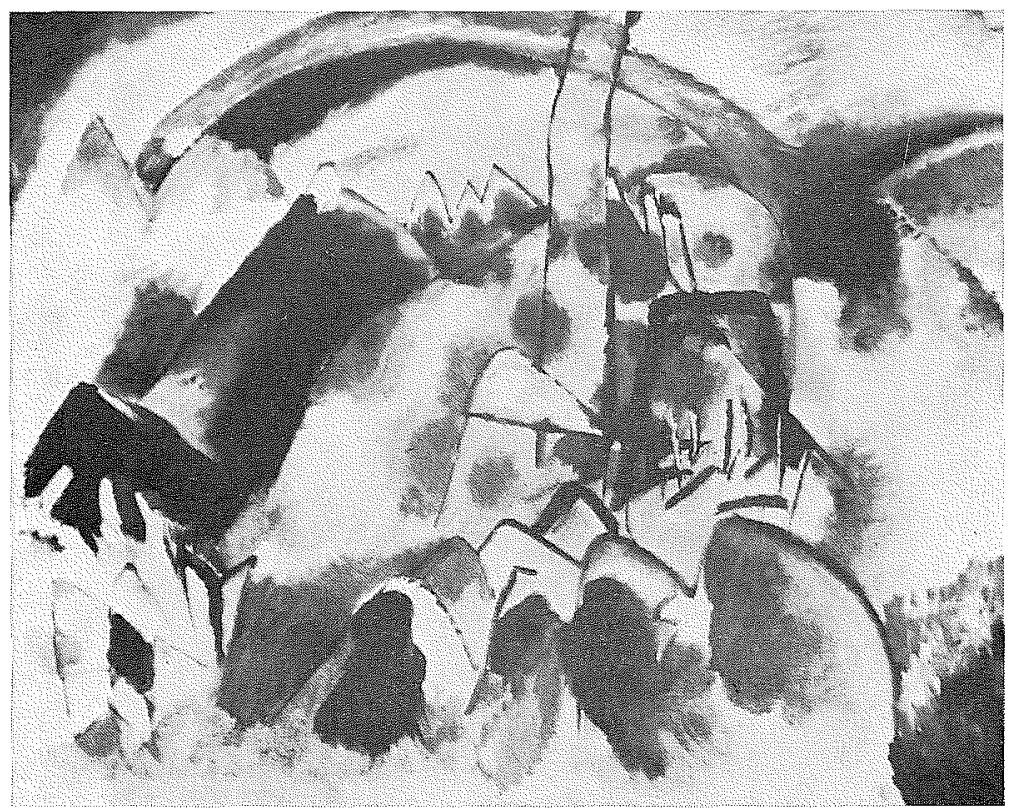

7. Kandinsky, Landschaft mit Kirche I (1913). Museum Folkwang, Essen.

Dinge zu den unsichtbaren zu erheben, von dem Körperlichen zu dem Geistigen. Denn dieses ist die Aufgabe der Bilder" 1.

Eine auffallende Parallele zu der ,bildlosekeit" der westlichen Tradition finden wir im Buddhismus. Es kann hier nicht die Rede davon sein, auf Feinheiten in der buddhistischen Meditation einzugehen, deshalb müssen wir uns damit begnügen einige charakteristische Züge im Schema hervorzuheben. Die sogenannten Dhyana- und Samadhi-Meditationen haben das Ziel den Sinn von der Welt der Formen zu der Welt der Formlosigkeit zu erheben $^{2}$. Die vier Bewusstseinszustände, die man Rupa Dhyanas nennt, erreicht man noch durch Meditation über sichtbare Formen, während die folgenden, höheren Stadien dagegen formlos, arupa, sind und Befreiung von dem Hindernis der materiellen Formen bewirken ${ }^{3}$. Im Zenbuddhismus, der Weiterentwicklung des Dhyana-Systems, wird die Formlosigkeit von entscheidender Bedeutung, und in der Zenkunst stossen wir sogar auf Bild-

\footnotetext{
${ }^{1}$ Johannes Gerson, Opera omnia, II, Strassburg 1514, fol. 7rM.

${ }^{2}$ Paravahera Vajiranāna Mahāthera, Buddhist Meditation in Theory and Practice, Colombo 1962, SS. 35 ff.

${ }^{3}$ Vajiranāna, SS, $332 \mathrm{ff}$.
} 


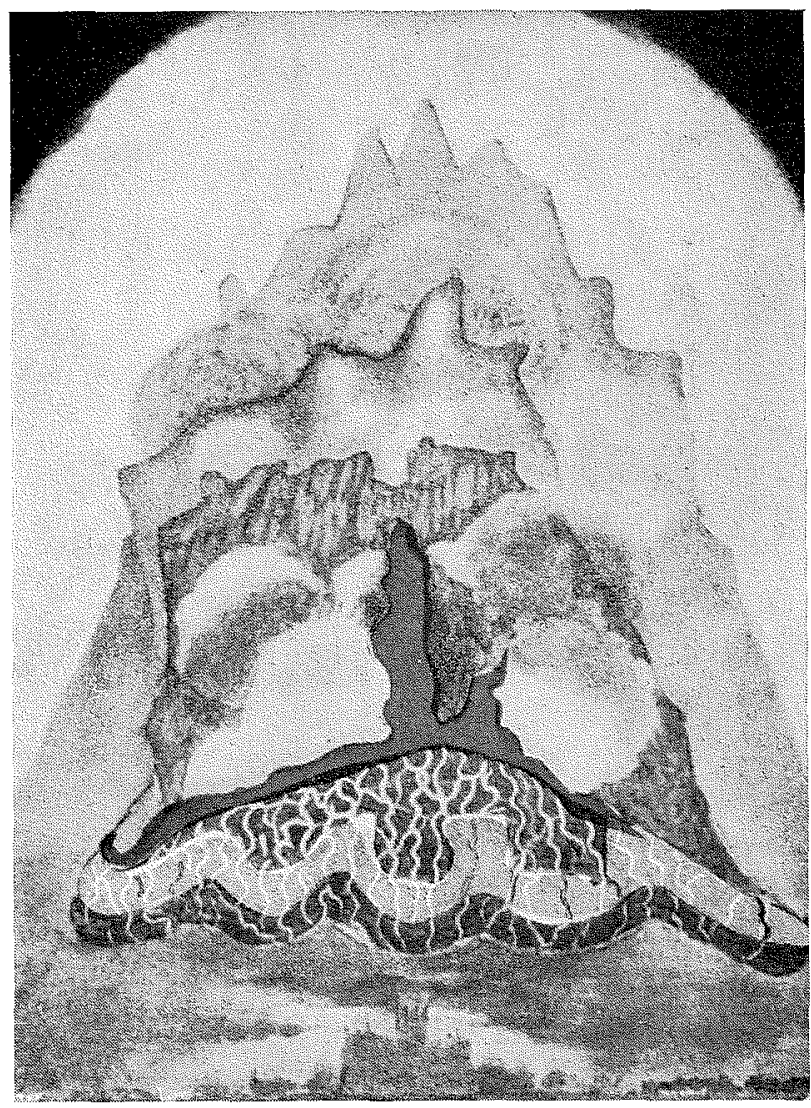

8. ,Wagner“. Aus Besant und Leadbeater, Tafel W.

darstellungen des Meditationsverlaufes, z. B. die Io-Bilder-Serie Das Ochsenhüten ${ }^{1}$.

In den Meditationsarten, die hier skizziert sind, findet sich also ein wichtiger gemeinsamer Zug: die Konzentration strebt nach einer Abstraktion von den sichtbaren Formen der materiellen Dinge und von bildlichen Vorstellungen. In der mittelalterlichen christlichen Mystik finden wir oft drei Stufen (Meditation, Spekulation, Kontemplation) ${ }^{2}$ in Übereinstimmung mit Augustinus' Unterscheidung von körperlichem, geistigem und intellek-

${ }^{1}$ Daisetz Teitaro Suzuki, Manual of Zen Buddhism, London 1956, SS. 127 ff. mit Abbildungen.

2 E. Benz, , ,Christliche Mystik und christliche Kunst", Deutsche Vierteljahrsschrift fïr Literaturwissenschaft und Geistesgeschichte, 12, 1934, SS. 22-48. 


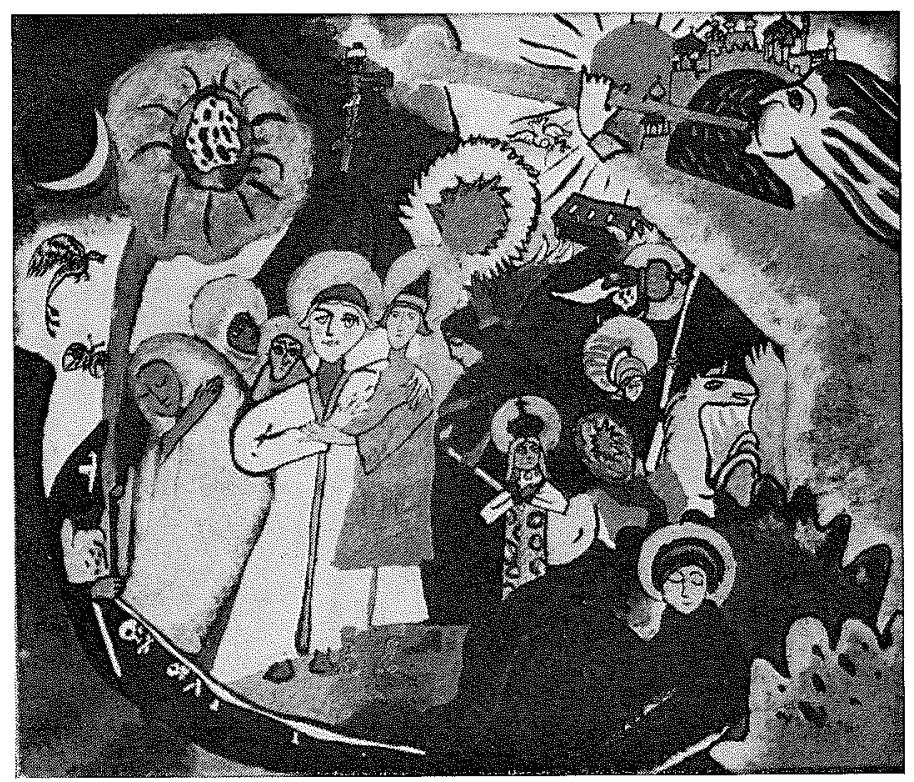

9. Kandinsky, Allerheiligen (I9I I). Städtische Galerie, München.

tuellem Schauen. Im Buddhismus ist bekanntlich die Einteilung bis zu grosser Verfeinerung getrieben. Beim Buddhismus und Neuplatonismus kommt übrigens ein gemeinsamer, gnostischer Zug hinzu: die höchsten, formlosen Stufen liefern eine konkrete und objektiv wahre Erkenntnis von der Beschaffenheit der Welt. Mit anderen Worten: die geistige Welt ist in Hinsicht auf das Materielle formlos. Im Buddhismus stellt man sich vor, dass diese Welt aus einer Art von Materie besteht, die viel feiner ist als der grobe Stoff der physischen Natur.

\section{Gegenstandslosigkeit in der abstrakten Kunst}

In den Jahren I9ro-I9I4 entstand in der Malerei die Richtung, die man unter dem Namen „abstrakte“, „,nonfigurative“ oder ,gegenstandslose“ Kunst versteht. In diesen Jahren malten der Franzose Robert Delaunay, der Tscheche František Kupka und der Holländer Piet Mondrian in Paris, und der Russe Wassily Kandinsky in München die ersten gegenstandslosen Bilder, d. h. Werke, bei denen alle abbildenden Züge ausgelassen sind, die aber auftreten mit dem Anspruch etwas anderes zu sein als Dekoration oder Ornament. 


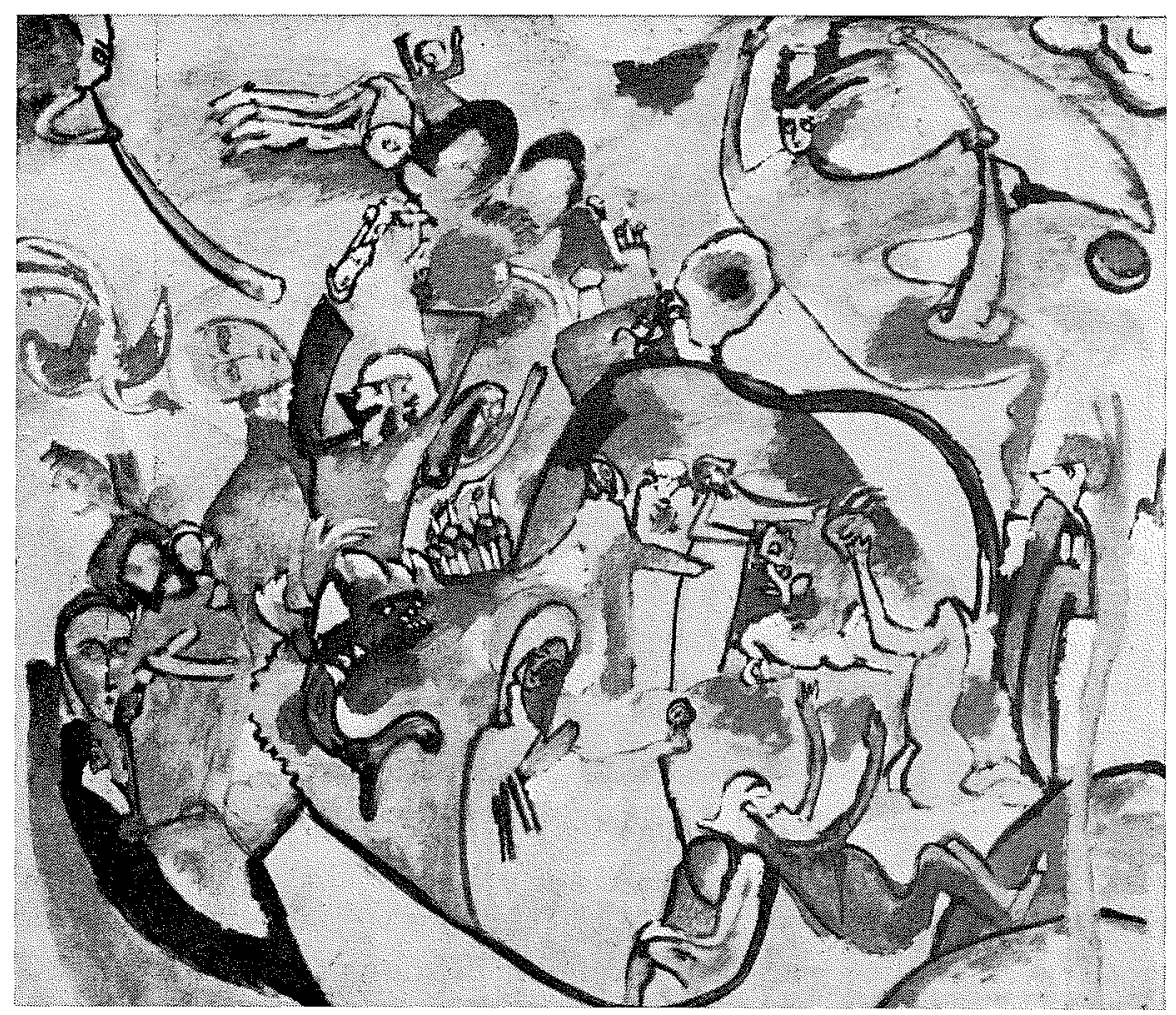

ıo. Kandinsky, Allerheiligen (19r I). Städtische Galerie, München.

Von diesen Künstlern ist wohl Kandinsky derjenige, der den grössten Einfluss ausgeübt hat, nicht zuletzt wegen seines umfassenden theoretischen Schrifttums. Der Titel seiner wichtigen Schrift von 1912, Über das Geistige in der Kunst ${ }^{1}$, deutet bereits an, was Kandinsky als die hauptsächliche Aufgabe der Kunst ansah, nämlich: einen geistigen Inhalt zu vermitteln. Naturalistische Kunst betrachtete Kandinsky als Ausdruck für die falsche und überwundene Weltanschauung des Materialismus², während ein neues, geistiges Zeitalter, eines der grössten in der Geschichte der Menschheit, sich in der abstrakten oder ungegenständlichen Kunst ankündiges. Für ein

${ }^{1}$ Wassily Kandinsky, Über das Geistige in der Kunst, München I912; hier zitiert nach der 7. Aufl. Bern 1963 und verkürzt $\ddot{U} G$.

${ }^{2}$ Kandinsky, $\ddot{U} G$ SS. 22, 73, I20 f., I34; ders., , Über die Formfrage“", Kandinsky \& Franz Marc (Hrsg.), Der Blaue Reiter, dokumentarische Neuausg. von K. Lankheit, München r965, SS. г32-137, г8г.

${ }^{3}$ Kandinsky, $\ddot{U} G$, S. 143, , Ưber die Formfrage“, S. I43. 


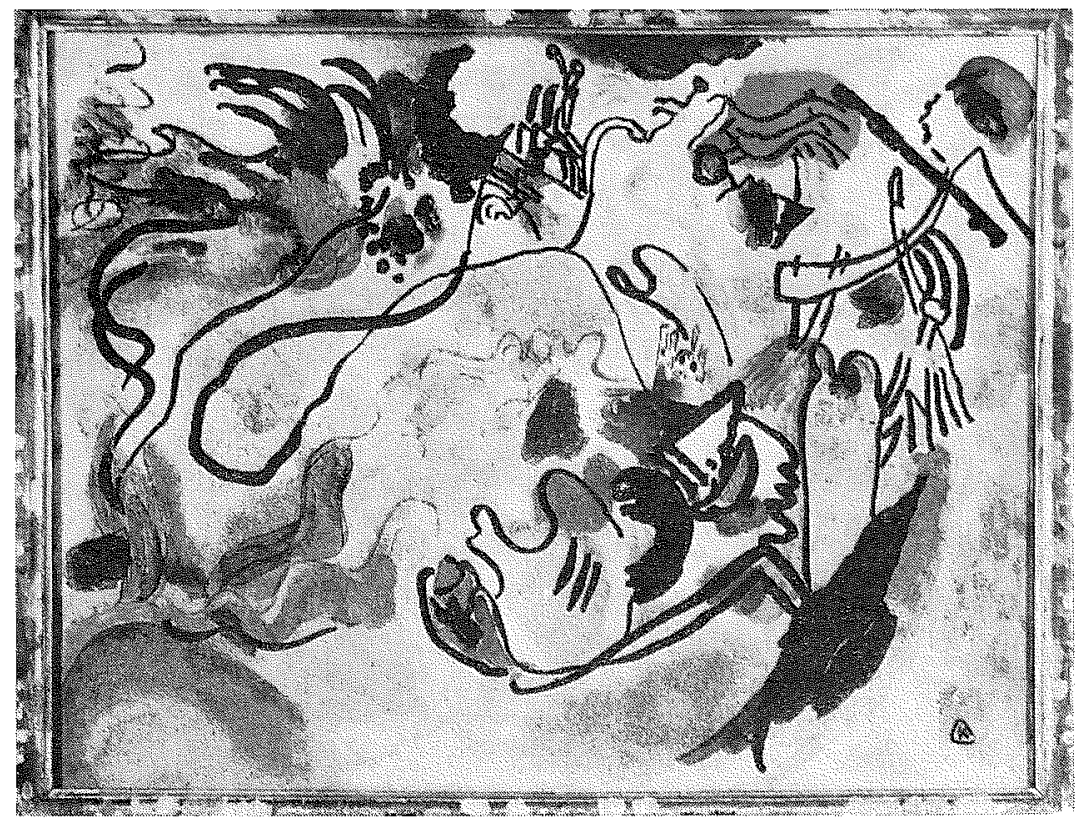

x r. Kandinsky, Fïngster Tag (r 1 r2). Sammlung Nina Kandinsky, Neuilly-sur-Seine.

Publikum das gewohnt ist, die gegenstandslose Kunst als einen Ausdruck von Ästhetizismus zu betrachten, kommt Kandinskys Betonen des Inhaltes vielleicht als eine Überraschung; wie kann man von Inhalt sprechen in einem Bild, das nichts vorstellt, möchte man sich fragen. Die Erklärung liegt darin, dass Kandinsky der Ansicht war, alle wahre Kunst habe einen geistigen Inhalt, der unabhängig ist von dem, was sonst dargestellt wird. Der Inhalt ist das Primäre und die Form sekundär, da sie zur Materie gehört. Darum gibt es im Prinzip kein sogenanntes Formproblem². Die materiellen Formen kann man weglassen so dass der geistige Inhalt rein hervortritt. Die äusserste Konsequenz wird so ein Bild, das ganz befreit ist von den materiellen Formen der physischen Wirklichkeit.

Ähnliche Gedankengänge treten auch bei Piet Mondrian auf. Mondrian meinte ,dat de geest beter benadert wordt door een vorm die korter bij den Geest is “" ${ }^{\text {" }}$ Die Theorie der abstrakten Kunst zeigt also grosse Ähnlichkeit mit den oben geschilderten Vorstellungen der Mystiker — in beiden Fällen

\footnotetext{
1 Kandinsky, , U'ber die Formfrage“, S. × $37, x 62$.

2 Seuphor, Mondrian, Reproduktion S. I Io eines Notizbuches von I910.
} 


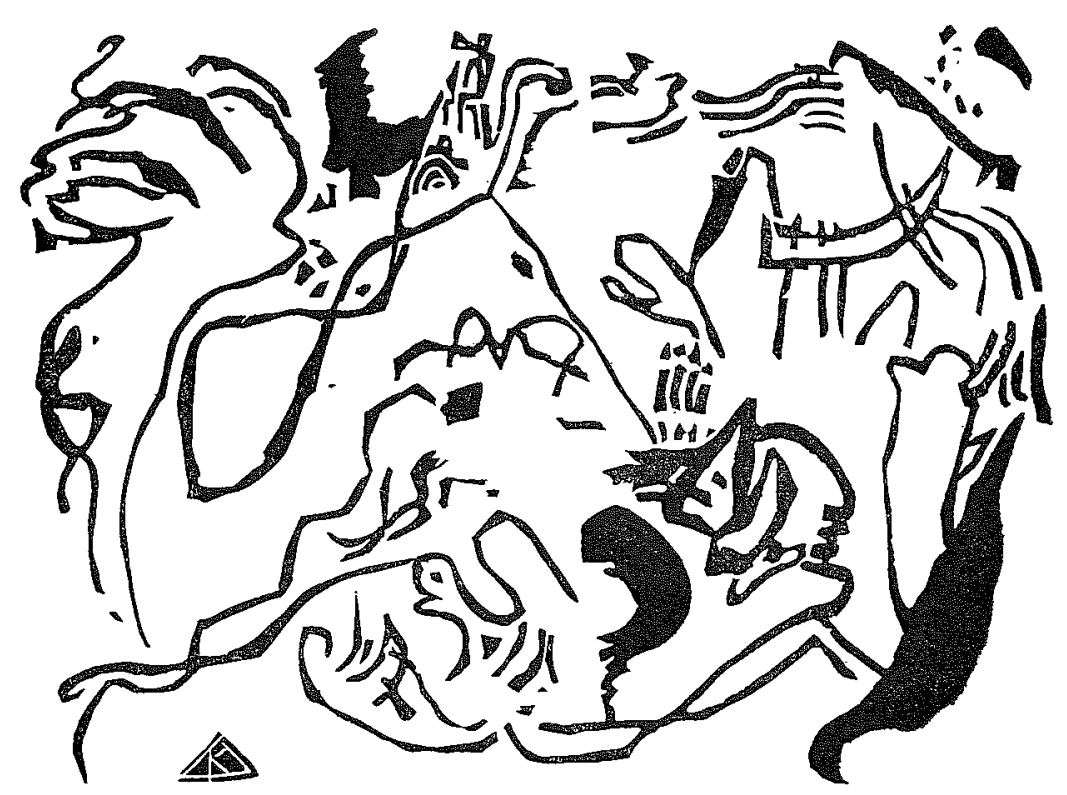

I2. Kandinsky, fïngster Tag. Holzschnitt aus Klänge (r9r2).

wird der von den Abbildern der Dinge befreite Zustand als die höchste Stufe dargestellt. Auch Kandinsky betrachtete die Abstraktion als einen Prozess, der in verschiedenen Stufen verläuft, und rechnete seinerseits mit drei Arten von Malereien:

I. direkter Eindruck von der ,,äusseren Natur", welcher in einer zeichnerischmalerischen Form zum Ausdruck kommt. Diese Bilder nenne ich ,Impressionen";

2. hauptsächlich unbewusste, grösstenteils plötzlich entstandene Ausdrücke der Vorgänge inneren Charakters, also Eindrücke von der ,,inneren Natur". Diese Art nenne ich ,,Improvisationen";

3. auf ähnliche Art (aber ganz besonders langsam) sich in mir bildende Ausdrücke, welche lange und beinahe pedantisch nach den ersten Entwürfen von mir geprüft und ausgearbeitet werden. Diese Art Bilder nenne ich ,Komposition“" Hier spielt die Vernunft, das Bewusste, das Absichtliche, das Zweckmässige eine überwiegende Rolle. Nur wird dabei nicht der Berechnung, sondern stets dem Gefühl recht gegeben ${ }^{1}$.

Die Kompositionen stellen offensichtlich die höchste Stufe dar, und dieser neue Geist des Kompositionellen steht nach Kandinsky im direkten $\mathrm{Zu}$ sammenhang mit dem kommenden, neuen Reiche, ,da dieser Geist die

${ }^{1}$ Kandinsky, $\ddot{U} G$, S. $142 \mathrm{f}$. 


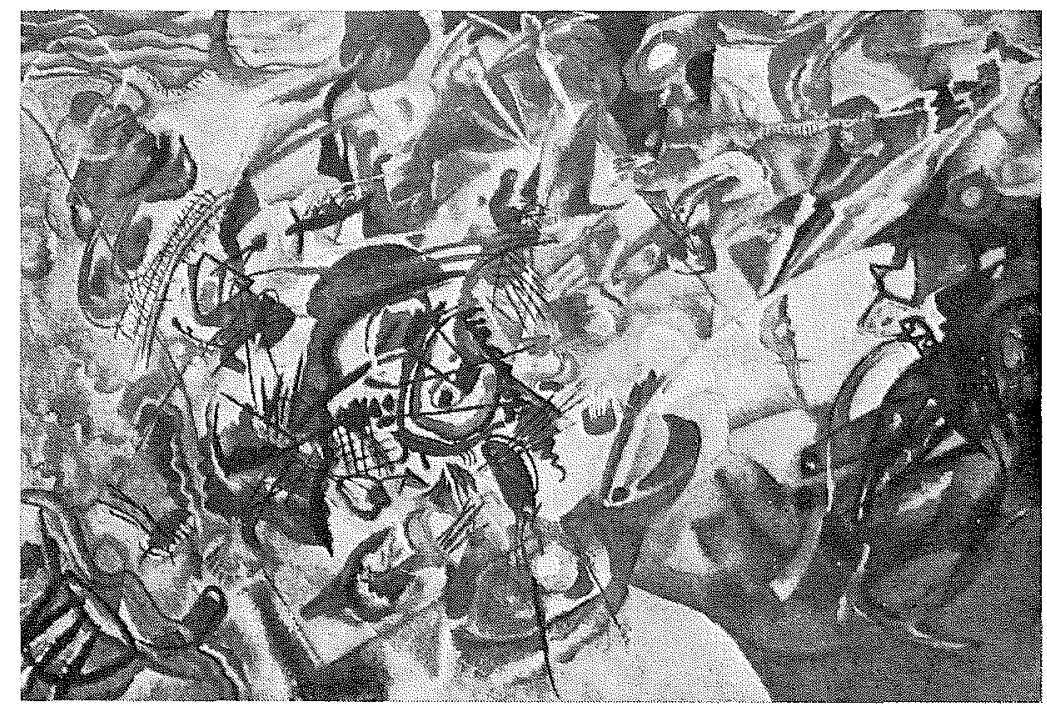

I3. Kandinsky, Komposition VII. (1913). Staatl. Tretjakow Galerie, Moskau.

Seele ist der Epoche des Grossen Geistigen". Will man den Vergleich vollständig machen, so muss man hinzufügen, dass Kandinsky auch glaubte, die Zukunft würde zeigen, dass seine gegenstandslosen Bilder tatsächlich naturgetreuer seien als frühere, äusserlich betrachtet, vorstellende Kunst (siehe unten S. 19). Die gegenstandslose Kunst ist ein Erkenntnisweg zu den kosmischen Gesetzen ${ }^{1}$. Die Gegenstandslosigkeit der abstrakten Kunst ebenso wie die Formlosigkeit des Neuplatonismus und die Arupa-Region des Buddhismus repräsentieren also eine objektiv wahre Wirklichkeit.

\section{Der historische Zusammenhang}

Damit sind wir angekommen bei der Frage nach dem geschichtlichen Verhältnis von der „Form- oder Bildlosigkeit“ der Mystik auf der einen Seite und der "Gegenstandslosigkeit" der abstrakten Kunst auf der anderen. Die Analogie ist so auffallend, dass sie kaum als etwas Zufälliges betrachtet werden kann. Eine mögliche Erklärung wäre, dass wir in beiden Fällen mit einer psychologisch bedingten Ähnlichkeit in der Zielsetzung zu tun haben, d. h., dass das für beide Phänomene gemeinsame Streben nach Reinheit

\footnotetext{
${ }^{1}$ Interview mit Kandinsky 1937; siehe Kandinsky, Essays über Kunst und Künstler, 2. Aufl., Bern $x_{96} 63$, S. 213.
} 
einen gleichartigen aber, geschichtlich gesehen, voneinander unabhängigen Ausdruck gesucht hat. Eine nähere Untersuchung zeigt aber, dass ein nachweisbarer geschichtlicher Zusammenhang zwischen den beiden besteht.

Es ist kaum ein Zufall, dass gerade die Pioniere der gegenstandslosen Kunst sich für Mystik interessierten. „Die meisten Maler sind nur Seher, während sie doch Hellseher sein sollten“, sagte Robert Delaunay'. František Kupka trat um die Jahrhundertwende in Paris als spiritistisches Medium auf und hatte starkes Interesse für Theosophie. Zur selben Zeit fand auch Piet Mondrian den Weg zur Theosophie und wurde I909 Mitglied der Theosophischen Gesellschaft. Mondrians Harmonielehre war beeinflusst von ausführlichen Diskussionen mit dem holländischen Theosophen Schoenmaekers ${ }^{2}$. Er stellte ausdrücklich Kunst und Meditation auf dieselbe Stufe und gab zwei Wege an zum Geistigen:

Zwei Wege zum Geistigen: der Weg der Lehrunterweisung (Meditation usw.), und der lange und sichere Weg der Evolution. Das offenbart sich in der Kunst. Man sieht in der Kunst das langsame Wachstum zum Geistigen hin, während die Künstler selbst sich dessen nicht bewusst sind $d^{3}$.

Von Kandinsky wiederum wissen wir, dass er in den entscheidenden Jahren um I9I o Meditationsübungen machte; wie es heisst ,nach indischen Vorbildern"4. Im Über das Geistige in der Kunst spricht er mit grosser Achtung von den Indern und von den ,rätselhaften Tatsachen“, die sie mit ihren uralten, halb vergessenen Methoden den zweifelnden Gelehrten unserer Kultur vorgelegt haben. Er meint, Madame Blavatsky gebühre die Ehre, als die erste ein festes Band zwischen den Indern und unserer Kultur gebunden zu haben, und er findet, dass die uralten Erkenntnismethoden, die die Theosophen wieder zum Leben gebracht haben, von Dr. Rudolf Steiner ,in eine verhältnismässig präzise Form gebracht" worden seien5. Kandinsky hatte im Jahre 1908 in Berlin Vorträge von Steiner besucht, und er besass auch mehrere von seinen Schriften. Notizen, die sich in der Gabriele-Münter-Stiftung der Städtischen Galerie in München befinden,

\footnotetext{
${ }^{1}$ Seuphor, Knaurs Lexikon, S. 50.

2 Jaffé, De Stijl, SS. 2 I f., 65-74, I22.

3 Seuphor, Mondrian, S. I 6 .

${ }^{4} \mathrm{~J}$. Eichner, Kandinsky und Gabriele Münter, von Ursprïngen moderner Kunst, München [1957], S. I9.

${ }^{5}$ Kandinsky, $\ddot{U} G$, S. $4 \mathrm{I}$ f.
} 
zeigen uns, dass Kandinsky die theosophische Meditationspraxis, wie sie dargestellt wird in Steiners Wie erlangt man Erkenntnisse der höheren Welten und Die Stufen der höheren Erkenntnis, gründlich durchgearbeitet hat und dass er sogar die Einzelheiten der von Steiner aufgestellten „Bedingungen“ und der ,praktischen Gesichtspunkte" aufgezeichnet hat ${ }^{1}$.

Dass Kandinsky direkt von Steiner theosophische und auf indischer und buddhistischer Mystik fussende Gedankengänge aufgenommen hat, kann also rein dokumentarisch belegt werden. Aber auch die Vorstellungen der westlichen Mystiker von der Bildlosigkeit der höheren Stufen hatten in Steiner einen Ausleger. In seinem Buch von I9or Die Mystik im Aufgange des neuzeitlichen Geisteslebens und ihr Verhältnis zur modernen Weltanschauung hatte Steiner die Entwicklung der Mystik von Eckhart bis Angelus Silesius geschildert. Hier zitiert er ausführlich Susos Ausspruch von der Bildlosigkeit der wahren Visionen (siehe oben) mit dem Zusatz; „Meister Eckhart lässt darüber keinen Zweifel, dass er die Anschauung ablehnt, die in körperlich-räumlichen Gebilden, ... das Geistige schauen will ““朔.

Wir wissen nicht ob Kandinsky gerade dieses Buch von Steiner gelesen hat. Aber wir wissen, dass er Steiners Schrift Die Stufen der höheren Erkenntnis studiert hat, und hier wird die westliche Mystik zusammengestellt mit einer orientalisch betonten Theosophie. In den Stufen wird die Erkenntnis in vier Stufen eingeteilt: ausser der alltäglichen sinnlichen Wahrnehmung (materielles Wissen) drei höhere Erkenntnisstufen, nämlich Imagination, Inspiration und Intuition. Auf der imaginativen Stufe ist die Welt schon so entmaterialisiert, dass Farben und Töne nicht mehr aufgefasst werden, als ob sie zu materiellen Gegenständen gehörten, sondern frei im Raume zu schweben scheinen. Oder wie es in Kandinskys Steiner-Referat heisst: „Das

\footnotetext{
1 Rudolf Steiner, Wie erlangt man Erkenntnisse der höheren Welten, Dornach $196 \mathrm{1}$, SS. 102, 90 (urspr. in Lucifer-Gnosis, Nr. I3-28, 1904-1905).

2 Steiner, Die Mystik, Dornach 1960, S. 74.

${ }^{3}$ Ringbom, „Art", S. 4I8. Siehe Steiner, Die Stufen der höheren Erkenntnis, Dornach 1959, S. 4I (urspr. in Lucifer-Gnosis, Nr. 29, 30, 32, 34, 35, Berlin 19051908): ,Zur Hervorrufung der imaginativen Erkenntnis aber kommt es gerade darauf an, zunächst Farben, Töne, Gerüche usw. zu haben die ganz losgelöst von allen Dingen 'frei im Raume Schweben' ", und S. 42 f.: ,, in der imaginativen Welt spricht alles so zum Menschen, wie wenn es unmittelbar intelligent wäre, während in der physischen Welt auch die Intelligenz nur auf dem Umwege durch die physische Körperlichkeit sich offenbaren kann".
} 
Schweben der Farben etc. 'ohne Grund u. Boden' (=ohne phys. Gegenstand) ist die Offenbarung der Wesenheiten, die den Menschen stets umgeben" "1. Auf der folgenden Stufe, der Inspiration, schreibt Steiner, ,,bleiben nun auch die Bilder weg“" ".Es sind gewisse Linienformen, Gestalten, die man erlebt ... Ja man fühlt das Ich als den Zeichner und zugleich als das Material mit dem gezeichnet wird ... Man lernt erkennen, dass man mit seinem bewegten Ich hineingeflochten ist in die schaffenden Weltenkräfte" ${ }^{2}$. Die höchste Stufe, die Intuition, wird auch in Steiners Geheimwissenschaft von I910 in folgender Weise geschildert: „Durch die Intuition ist der letzte Rest des Sinnlich-physischen von des Menschen Eindrücken abgestreift; die geistige Welt beginnt für die Erkenntnis offen zu liegen in einer Form, die nichts mehr gemein hat mit den Eigenschaften der physischsinnlichen Welt"،

Imagination, Inspiration, Intuition, die Steiner in seiner graphisch anschaulichen Prosa schildert, treten dann wieder auf in Kandinskys Einteilung in Impression, Improvisation, Komposition, wo ja die Komposition die höchste Stufe bildet und die Epoche des Grossen Geistigen ankündigt. Das Wort „Komposition“, schreibt Kandinsky 1913, wirkte auf ihn wie ein Gebet".

So bildet die Theosophie ein historisches Verbindungsglied zwischen Mystik und abstrakter Kunst. Die Theosophie hatte Lehrgut aufgenommen von spätantiker und westlicher Mystik sowie auch vom Buddhismus. Der buddhistische Unterschied zwischen der Welt der Formen (rupa) und der formlosen Welt (arupa) spielt eine wichtige Rolle in der Theosophie (vgl. Abb. I). Dass Kandinsky mit der Vorstellung von Rupa und Arupa vertraut war, dafür gibt es sichere Beweise. In seinem Buche Theosophie vom Jahre I908 schildert Steiner die sieben Regionen der geistigen Welt. Die drei höchsten, schreibt er, werden „Arupa-Abteilungen genannt, im Gegensatz zu den vier unteren, welche Rupa-Regionen heissen. Arupa ist 'formlos';

\footnotetext{
1 Steiner, Stufen, S. 20.

2 Ibid., S. 7 I.

${ }^{3}$ Steiner, Die Geheimzissenschaft im Umriss, Dornach r962 (I. Aufl. Leipzig r9ro), S. 369 .

${ }^{4}$ Kandinsky $I g \circ I-I 9 I 3$, Berlin 1913 , S. xiii.
} 
rupa ist 'geformt"'1. In Kandinskys eigenem Exemplar dieses Buches, welches jetzt in der Gabriele-Münter-Stiftung der Münchener Städtischen Galerie aufbewahrt wird, ist dieser Abschnitt reichlig angestrichen vom Künstler, der auch am Rande die sieben Regionen numeriert hat.

Der Gedanke einer feineren, höheren Materie, die nur für höhere Sinne zugänglich ist, wird auch von der Theosophie übernommen und überliefert. In ihrem Buch Gedankenformen beschreiben Annie Besant und C. W. Leadbeater die Phänomene, die sich in den höheren Regionen zeigen. Ausser dem materiellen, physischen Körper (den ja jeder sehen kann) hat der Mensch noch höhere Körper von feinerer Materie (die nur Hellseher wahrnehmen können). In den höheren Körpern von feinerer Materie können die Gedanken und Gefühle des Menschen beobachtet werden als ein Muster von Farben und Formen, die von denen der Sinneswelt gänzlich abweichen (Abb. 2-3). Aus einem höheren Körper kann sich ein Teil ablösen und dann haben wir eine sogenannte Gedankenform, eine Konfiguration, die ein selbständiges Wesen ist und die keine Verbindung mehr hat mit den physischen Dingen. Diese Gedankenformen bilden keine Formen ab, die zu dem physischen Plan gehören, sondern wir bekommen statt dessen, schreiben Besant und Leadbeater ${ }^{2}$, einen Einblick in bezug auf die Formen die den höheren Regionen eigen sind. So werden in dem Bild Bei einem Schiffbruch (Abb. 5) keine menschlichen Gestalten oder physischen Gegenstände wiedergegeben, sondern nur die geistigen Manifestationen der Personen, wahrnehmbar nur für Hellseher. Die Gedankenform rechts unten gehört zu einem Passagier, der von Panik ergriffen ist. Von Panik zeugt auch die Form unten links, aber hier hat der Passagier zu beten angefangen, was man ablesen kann an der blaugetönten Spitze die an dem oberen Teil hervorragt. Einen Kontrast zu diesen beiden bildet die Form zu oberst, die zu dem Kapitän gehört diese Form drückt Selbstvertrauen und Entschlossenheit aus. Gelbe Farbtöne zeigen, dass sein Intellekt an einer Lösung des Problems arbeitet; das Grüne zeigt Sympathie für die Verunglückten, und die scharfe rote Spitze den Ärger darüber, dass das Unglück geschehen ist.

Kandinsky besass die deutsche Ausgabe von Besant und Leadbeaters

\footnotetext{
1 Steiner, Theosophie, Einführung in übersinnliche Welterkenntnis und Menschenbestimmung, 2. Aufl., Leipzig 1908, S. 105.

${ }^{2}$ Annie Besant \& C. W. Leadbeater, Gedankenformen, Leipzig r 908, S. 38 f.
} 
Gedankenformen ${ }^{1}$ und kannte sicher auch Leadbeaters Der sichtbare und der unsichtbare Mensch ${ }^{2}$, das die feinmateriellen Körper beschreibt und illustriert. In den theosophischen Texten und Illustrationen fand Kandinsky eine vielleicht etwas grobe aber doch lebhafte und suggestive Darstellung des Geistigen. Genau betrachtet sind ja diese theosophischen höheren Welten nicht formlos; dann wären sie ja leer, wie in der oben erwähnten Zenserie, hingegen sind sie unabhängig von den Formen des physischen Planes³. Das überzeugte Kandinsky von der Berechtigung, die in materieller Beziehung vorstellende oder realistische Kunst zu lassen. Diese Kunst ,,sucht ihren inhaltlichen Stoff in der harten Materie, da sie die feine nicht kennt. Die Gegenstände, die wiederzugeben sie für ihr einziges Ziel hält, bleiben unverändert dieselben. Das 'Was' in der Kunst fällt eo ipso aus. Nur die Frage, 'wie' derselbe körperliche Gegenstand zum Künstler wiedergegeben

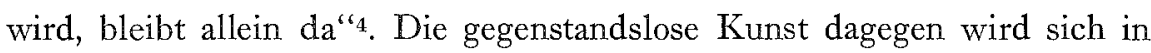
der Zukunft als wahrer, naturgetreuer erweisen als die frühere Kunst ${ }^{5}$.

Wie sich Kandinsky die Einzelheiten der formlosen Welt der Theosophie zu eigen machte, geht aus einem Vergleich einiger seiner Werke aus der Zeit hervor, von der hier die Rede ist, mit den Illustrationen in Besants und Leadbeaters Büchern. Die Improvisation Ig (IgII, Abb. 4) zeigt eine für diese Periode beachtenswerte Emanzipation von Farben und Formen von ihrer gegenseitigen Abhängigkeit und von der Struktur der abgebildeten Gegenstände. Das Bedeutungsvolle ist, dass die Umrisse keine bestimmten

1 Das Buch befindet sich jetzt in der Bibliothek Kandinskys in Neuilly-sur-Seine. Vgl. auch Grohmann, SS. 4I, 108,

${ }^{2}$ C. W. Leadbeater, Der sichtbare und der unsichtbare Mensch, Leipzig rgo8. Gedankenformen enthält mehrere Hinweise auf Der Sichtbare usw., und darum scheint es berechtigt anzunehmen, dass Kandinsky auch mit dem letzteren Buch vertraut war.

3 Nach theosophischer Auffassung ist diese Formlosigkeit relativ. In einer Vortragsserie, die I902 auf deutsch herauskam, erklärt Annie Besant: ,, Alle höheren Welten sind 'formlos', wenn sie von unten betrachtet werden, d. h. von Wahrnehmungsmitteln, die für den Gebrauch der niederen Welt eingerichtet sind; wenn aber ein Mensch die Fähigkeit entwickelt hat, auf die Schwingungen irgend einer höheren Welt zu antworten, dann ist sie für ihn eine Welt der Form und nicht der Formlosigkeit" (Die Entwicklung des Lebens und der Form, Berlin 1902, S. 139). Für Kandinskys Benützung des Begriffs „Schwingung“ oder „Vibration“, siehe Ringbom, ,Art", S. $400 \mathrm{f}$.

${ }^{4}$ Kandinsky, $\ddot{U} G$, S. 32 .

${ }^{5}$ Kandinsky, Essays, S. 152. 
Farbflächen mehr umschliessen; statt dessen sind die Figurenmotive sozusagen von ihrem Inhalt entleert worden und die Farben fliessen aus in einem freien Muster. Gerade so sollten ja nach theosophischer Anschauung die Farben sich zeigen auf den höheren Stufen der Erkenntnis; gleichzeitig mit dieser Auflösung kommen auch neue Farben hinzu, welche die Gedanken und Gefühle der betrachteten Personen ausdrücken. In der Improvisation I9 ist von den Figuren der Gruppe rechts nur die Konturschale übrig als eine Andeutung an die materielle Form der Gestalten, genau so, wie in den theosophischen Illustrationen der Ort des physischen Leibes summarisch durch eine Konturzeichnung angedeutet wurde (Abb. 2-3).

Dieselbe Entmaterialisierung kann auch in Kandinskys Landschaften beobachtet werden. Die Landschaft von I9Io (Abb. 6) gibt noch immer deutlich die Kontur wieder, und ist tatsächlich zurückzuführen auf eine am Ort ausgeführte kleine Ölskizze. Die Variation von I9I3 (Abb. 7) zeigt dagegen einen höheren Grad von Abstraktion. Auf diesem späteren Bild sehen wir wieder, wie die Farben sich gelöst haben von den Dingen und frei zwischen den Formen zu schweben scheinen. In den verschiedenen Fassungen desselben Motivs, die Kandinsky in den Jahren I910-1913 ausführte, können wir ausserdem eine fortlaufende Annäherung an eine der Illustrationen in Besants und Leadbeaters Gedankenformen beobachten, nämlich ein Bild, das eine Form vorstellt, die nicht direkt durch einen Gedanken hervorgebracht wurde, sondern durch die Aufführung eines Musikstückes (Abb. 8)1. Ein Vergleich zwischen der musikalischen Landschaft und Kandinskys Bildern zeigt sogar direkte Übereinstimmungen in Einzelheiten: der durchgehend gelbliche Ton des Hintergrundes, dessen violette Bergspitzen, der diffuse blaue Farbfleck in der Mitte usw.

Die endgültige Durchführung der Abstraktion und Entmaterialisierung kann schliesslich in einer Reihe von apokalyptischen Bildern verfolgt werden.

Ein Allerheiligenbild von I9I I (Abb. 9) zeigt einen von der Volkskunst inspirierten Reichtum an Einzelheiten, der in einem Ölgemälde von I9II ,entmaterialisiert" wurde (Abb. Io). Die Motive sind immer noch voll erkennbar: Engel oben in den Ecken, in der Mitte ein Hügel mit Gebäuden

${ }^{1}$ Besant \& Leadbeater, Gedankenformen, SS. $108 \mathrm{ff}$. 
und Türmen, links der hl. Wladimir, im Himmel Elias mit dem Wagen, im Vordergrund Gruppen von Figuren, Tote, die auferstehen, Ungeheuer usw. Das Hinterglasgemälde füngster Tag von Igı2 (Abb. II) wäre schon schwer zu verstehen ohne die Hilfe des Vorangegangenen, und die Holzschnittfassung in Kandinskys Gedichtsammlung Klänge (r9r3, Abb. 12) setzt unbedingt die Kenntnis der vorhergegangenen Versionen voraus, um verständlich $z u$ sein $^{1}$.

Man kann mit grosser Gewissheit sagen, dass die monumentale Komposition VII (r913, Abb. I3), der Höhepunkt in Kandinskys Schaffen aus dieser Zeit, auf ein apokalyptisches Thema zurückzuführen ist, aber hier hat der Künstler endgültig die Verbindung mit den gegenständlichen Formen abgebrochen. Dies ist ein ,reines“ Bild, eine Komposition, und kann also nach Kandinskys Einteilung gesehen werden als ein Ausdruck für die erwartete „Epoche des Grossen Geistigen“.

Noch ein Jahrzehnt später, I922, sprach Kandinsky in einem Gespräch mit Lothar Schreyer Gedanken aus, die darauf hindeuten, dass er immer noch die gegenstandslose Kunst als eine immaterielle Verkündigung eines geistigen Inhalts und als Ausdruck für eine kommende geistige Epoche betrachtet. Bei dieser Gelegenheit wies Kandinsky auch ausdrücklich auf die Mystik und auf ,einen neuen religiösen Kult" hin.

Erschrecken Sie nicht, wenn ich Ihnen jetzt sage, dass ich meine eigenen Bilder, besonders seit ich ungegenständlich male und je älter ich werde, als christliche Bilder erkenne. Das ist mir vor zehn Jahren unabweisbar deutlich geworden ${ }^{2}$.

Ich sehe das Reich des Geistes im Lichte heraufziehen und will es, soweit ich es mit meiner Kunst vermag, verkünden. Darum male ich keine Christusbilder, male nicht den Menschensohn, der menschlich darstellbar ist. Der Heilige Geist ist nicht gegenständlich zu erfassen, sondern nur ungegenständlich. Das ist mein Ziel: Licht vom Lichte, das fliessende Licht der Gottheit, den Heiligen Geist zu verkünden. Vermögen die Menschen die Verkündigung aufzunehmen, das Licht zu schauen? Wann?

\footnotetext{
${ }^{1}$ Für die ,,Vergeistigung" dieses Motifs, siehe H. K. Röthel in: Vasily Kandinsky, Painting on Glass (Hinterglasmalerei), Anniversary Exhibition, The Solomon R. Guggenheim Museum, New York i966, S. i I f.

${ }^{2}$ Lothar Schreyer, Erinnerungen an Sturm und Bauhaus, München I957, S. 232 f.

${ }^{3}$ Schreyer, S. 235.
} 